\section{Corticium praticola Kotila ; an Interesting Basidiomycete occurring in England}

THE usual forms of Rhizoctonia when grown on agar culture remain sterile, and are differentiated mainly on the basis of vegetative characters. During a study of the occurrence of Rhizoctonia solani Kuhn in England, a number of isolates falling within the limits of the form genus Rhizoctonia regarding vegetative characters formed basidiospores freely on agar cultures. These isolates were afterwards identified as Corticium praticola Kotila ${ }^{1}$, although the measurements of basidia, sterigmata and spores were all slightly larger than those given by Kotila.

This is the first record of this fungus from Great Britain, and the first confirmatory record of Kotila's original work. D. P. Rogers ${ }^{2}$, in dealing with the genus in 1943, failed to accept the species due to absence of original material and lack of confirmatory isolations.

The fungus was obtained from four widely separated localities in England. At Slough and Bristol, soil was very heavily infested with the organism, and at Cheshunt and Leeds it was fourud causing foot rot of cucumber and rot of dahlia petioles and leaves respectively. It is probably of common occurrence and may have been isolated previously not only in England but also in other countries and included under $R$. solani. As supporting evidence for this suggestion, isolates closely agreeing with the English ones have been obtained from several soils in South Australia during 1951. Although these isolates have not yet been induced to form basidiospores, they have been temporarily classified as C. praticola.

$C$. praticola differs from $R$. solani in its thinner hyphal diameter $(6-9 \mu, C$. praticola ; 8-12 $\mu, R$. solani $)$, faster growth-rate $(0.55 \mathrm{~mm}$. per hour, $C$. praticola; less than $0.5 \mathrm{~mm}$. per hour, $R$. solani), and by the white mealy appearance of its mycelium later turning greyish-brown, in contrast to the felt-like cinnamonbrown of $R$. solani.

C. praticola is pathogenic to sugar beet seedlings above $15^{\circ} \mathrm{C}$. and to a wide variety of hosts, including lettuce, tomato, cabbage and dahlia, at temperatures of $23^{\circ}-25^{\circ} \mathrm{C}$. and above. This fact, together with its vigorous saprophytic growth, makes it a potential danger particularly if it becomes established in vegetable-growing areas. In South Australia the organism has caused severe losses of tomato seedlings being raised under warm conditions in nurseries.

As a laboratory organism, $C$. praticola is useful for teaching purposes. When grown on a soil extract agar with 0.1 per cent added sugar, the fruiting structures can be clearly demonstrated with little visual interference from sterile hyphæ. In view of its close similarity to $R$. solani, it may also prove a useful tool in aiding our understanding of the various isolates of $R$. solani and their relationship with Corticium solani.

The fungus was isolated while working in Prof. W. Brown's laboratory at the Imperial College of Science and Technology, London.

\section{Waite Agricultural Research Institute,}

\section{N. T. FuentJe}

University of Adelaide. April 17.

${ }^{1}$ Kotila, J. E., Phytopath., 19, 1059 (1929)

${ }^{2}$ Rogers, D. P., Farlowia, 1, 95 (1943).

\section{Isolation of Triploid Saccharomyces cerevisiz}

THE production of tetraploids in a brewery yeast has been reported by Subramaniam and Krishna Murthy $^{1}$ and Mitra and Subramaniam ${ }^{2}$, following treatment with acenaphthene and chrysene. These authors did not present genetic evidence to support their claim, which was based mainly on colony and other gross observations. Cytological evidence has recently been presented for a tetraploid with four chromosomes, derived from a "diploid two chromosome ... strain"s. Tobias ${ }^{4}$ studied a yeast isolated after acenaphthene treatment in terms of survival after X-irradiation, and concluded that the isolate was tetraploid. DeLong and Lindegren ${ }^{5}$ have characterized certain strains of Saccharomyces cerevisioe as triploid and tetraploid, based on the shapes of survival curves after ultra-violet irradiation. A paper by Lindegren and Lindegren ${ }^{6}$ has recently appeared, describing the production of tetraploid yeast by crosses of diploids homozygous for complementary mating types. We wish to give a preliminary report on a procedure for the isolation of pure triploid clones of $S$. cerevisice.

A diploid clone was isolated (by the method of Pomper and Burkholder ${ }^{7}$ ) which required adenine and uracil for growth, that is, was homozygous for recessive alleles for adenine and uracil syntheses. This diploid was heterozygous for mating type ( $a$ and $\alpha$ of Lindegren and Lindegren ${ }^{8}$ ), and for genes involved in the syntheses of methionine and tryptophane. Haploid clones of both mating types, requiring methionine and tryptophane for growth (the same loci for which the diploid was heterozygous), were available to us. The diploid was mixed with both haploids (in separate tubes) by the 'mass mating' procedure of Lindegren and Lindegren". After incubation for three days at $25^{\circ} \mathrm{C}$., the cells were washed and plated on agar containing carbohydrate, nitrogen, vitamins and buffer, but lacking adenine, uracil, methionine and tryptophane. Under these conditions, neither the haploids nor the diploid should be able to grow, and, indeed, control plates had no colonies. However, a few colonies appeared on the plates from both 'crosses'. These were isolated as presumptive triploids. Since it was possible that we had merely isolated either doubly reverted diploids or haploids, it was necessary to carry out further tests to evaluate the assumption that these isolates were indeed triploids.

Neither the haploids nor the diploid employed in these, crosses could themselves sporulate under our test conditions. The observation that the 'triploid' isolates sporulated lent support to the belief that the test isolates were, at least, different from the original components of the cross. Dissections were carried out on asci from the 'triploids' arising from both crosses. Examination of the mating-type behaviour of the ascospore isolates revealed that a number of them did not conjugate with tester stocks of either mating type.

In one ascus, two of the four spores mated, and two did not. The latter pair were found to sporulate; the former did not. The sporulating ascospores behaved as typical diploids and segregated for mating type, thus explaining the failure of the parental spores to mate. The conclusion appears justified that the parent culture had produced two haploid and.two diploid clones, and further, that the parent was probably a triploid or its nuclear equiv- 